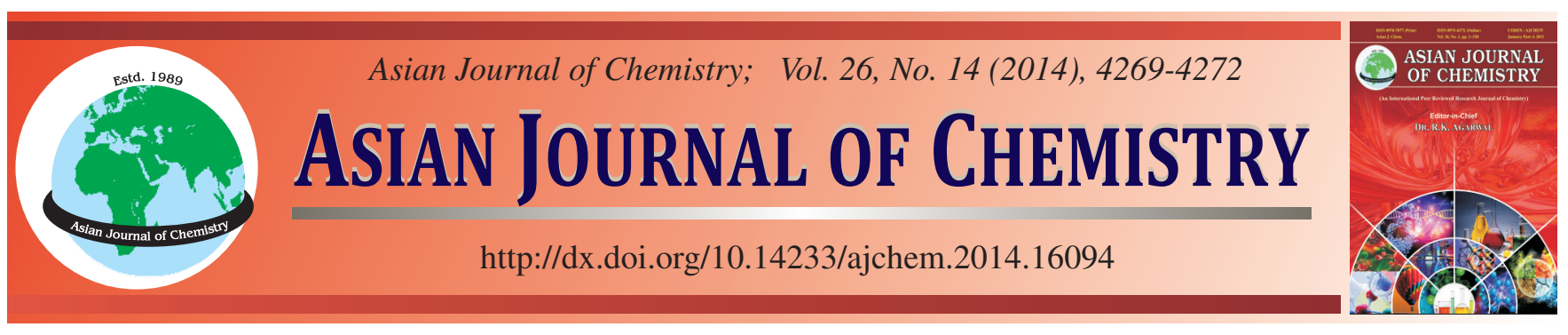

\title{
J- and H-Aggregation of 5,10,15,20-Tetrakis-(4-sulfonatophenyl)porphyrin within Stimuli Responsive Complex Micelles via Electrostatic Interaction
}

\author{
Qingyuan $\mathrm{YU}^{2}$, Chenglin Wu ${ }^{1, *}$ and FANG WANG ${ }^{1}$
}

\begin{abstract}
${ }^{1}$ School of Pharmaceutical and Chemical Engineering, Taizhou University, Taizhou 317000, Zhejiang Province, P.R. China
\end{abstract}
${ }^{2}$ First Middle School of Taizhou City, Taizhou 317000, Zhejiang Province, P.R. China

*Corresponding author: E-mail: clwu@tzc.edu.cn

\begin{abstract}
In aqueous solution, complex micelles were prepared by micellization of 5,10,15,20-tetrakis-(4-sulfonatophenyl)porphyrin (TPPS) and poly(2-(dimethylamino)ethyl methacrylate)- $b$-poly $(N$-isopropylacrylamide) (PDMAEMA- $b$-PNIPAM) at $\mathrm{pH}<7$ based on electrostatic interaction. The resultant complex micelles had a core-shell structure with complex TPPS/PDMAEMA as a core and PNIPAM as a shell. The anionic TPPS in the micellar core formed J-aggregates ( $\mathrm{pH} 1.5)$ and $\mathrm{H}$-aggregates $(\mathrm{pH} 3-\mathrm{pH}$ 7) due to electrostatic interaction with positive charged PDMAEMA. When the temperature was above the lower critical solution temperature (LCST) of PNIPAM, the thermoresponsive block collapsed on the complex micellar core, while the aggregation form of TPPS remained unchanged.
\end{abstract}

Keywords: Core-shell, 5,10,15,20-tetrakis-(4-Sulfonatophenyl)porphyrin, J-Aggregates, H-Aggregates.

\section{INTRODUCTION}

Nanostructure materials with tunable morphology have attracted considerable interest over the past decades because of unique architectures, tailored physicochemical properties, central roles in fabricating nanoelectronics and potential applications in bionanotechnology. Self-assembly is a ubiquitous principle in nature, which can lead to ordered architectures and it is currently considered to be an efficient strategy for manufacturing ordered nanostructures ${ }^{1}$. As natural pigments, porphyrins can spontaneously self-assemble into ordered nanostructures through noncovalent interactions, which are potential applications in photoelectronic and nonlinear optical devices ${ }^{2}$. It is worth noting that the aggregation of prophyrins has a deep impact on many physicochemical properties of these molecules. 5,10,15,20-tetrakis-(4-sulfonat-ophenyl)porphyrin (TPPS) has been well investigated because of its ability to form J-aggregates (side-by-side) and $\mathrm{H}$-aggregates (face-toface) under certain concentration, $\mathrm{pH}$ and ionic strength ${ }^{3}$. So far, a wide variety of sophisticated TPPS-containing nanomaterials have been developed in the presence of ions, surfactant, biological substances and polymer templates ${ }^{4,5}$.

In selective solvents, block copolymers can self-assemble into micelles and mesophases of various morphologies above the critical micelle concentration ${ }^{6}$. Among them, stimuliresponsive double hydrophilic block copolymer (DHBC) represents a special case. Upon a proper combination of external stimuli such as temperature, $\mathrm{pH}$ and ionic strength, one block of DHBC can become insoluble, while the other block remains soluble ${ }^{7}$. Micellization of DHBC and TPPS have attracted considerable interest for it could be formed especial structure and property. Zhao et al. ${ }^{8}$ reported that $\mathrm{J}$ - and $\mathrm{H}$-aggregates of TPPS were formed in the complex micelles of TPPS/PEG- $b$ P4VP. Additionally, the pyridine aromatic units were apt to result in the monomeric form of TPPS at $\mathrm{pH}$ above $3.5^{9}$.

Herein, the aggregation of TPPS was investigated in the presence of thermo and $\mathrm{pH}$-sensitive poly(2-(dimethylamino) ethyl methylacrylate)- $b$-poly ( $N$-isopropylacrylamide) (PDMAEMA- $b$-PNIPAM) (Fig. 1). Complex micelles of TPPS/

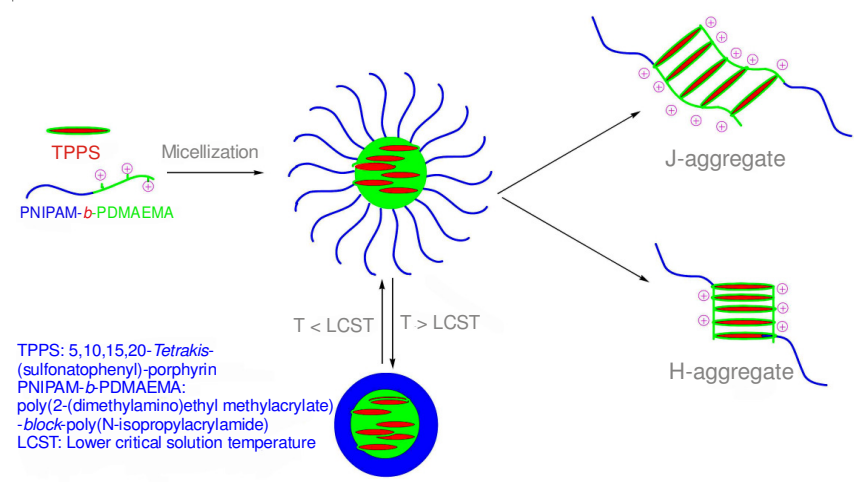

Fig. 1. Illustration of TPPS-induced micellization of PDMAEMA- $b$ PNIPAM and their $\mathrm{pH}$-responsive and thermo-responsive 
PDMAEMA- $b$-PNIPAM were formed through electrostatic interaction at room temperature and $\mathrm{J}$ - and $\mathrm{H}$-aggregates of TPPS were formed at different $\mathrm{pH}$ values. The morphology of the complex micelles was changed dramatically, when the solution temperature is higher than the lower critical solution temperature (LCST) of PNIPAM.

\section{EXPERIMENTAL}

Well-defined diblock copolymer PDMAEMA ${ }_{34}-b$-PNIPAM ${ }_{132}$ (PDI: 1.23), with the subscripts indicating the number of the repeating units, was synthesized by sequential reversible addition fragmentation chain transfer polymerization (RAFT) of DMAEMA and NIPAM with CPDB as chain transfer agent (CTA) and AIBN as initiator in 1,4-dioxane ${ }^{10}$. TPPS was purchased from Dojindo laboratory as an acid and used without further purification.

Preparation of the TPPS/PDMAEMA- $b$-PNIPAM complex micelles: The diblock copolymer PDMAEMA PD- $_{34}$ $\mathrm{PNIPAM}_{132}$ was first dissolve in $\mathrm{HCl}-\mathrm{KCl}$ buffer at $\mathrm{pH} 1.5$, $\mathrm{CH}_{3} \mathrm{COOH}-\mathrm{CH}_{3} \mathrm{COONa}$ buffer at $\mathrm{pH} 3$ and $\mathrm{pH} 5, \mathrm{Na}_{2} \mathrm{HPO}_{4}-$ $\mathrm{NaH}_{2} \mathrm{PO}_{4}$ buffer at $\mathrm{pH} 7$, with the polymer concentration $0.1 \mathrm{~g}$ $\mathrm{L}^{-1}$, respectively. All the buffer solutions had the same concentration of $0.1 \mathrm{~mol} \mathrm{~L}^{-1}$. The TPPS solution was prepared by dissolving in the neutral Milli-Q water (18 MO), stored in the dark and used within a day after preparation. The concentration of the TPPS solution $\left(28.5 \mu \mathrm{mol} \mathrm{L}^{-1}\right)$ was determined spectrophotometrically using $\varepsilon 414=5.33 \times 105 \mathrm{~L} \mathrm{~mol}^{-1} \mathrm{~cm}^{-1}$ at the soret maximum from an aliquot of the stock solution diluted $1 / 20$ by volume with $0.1 \mathrm{mM}$ phosphate buffer ${ }^{11}$. Micellization was carried out by adding the TPPS solution dropwise into the polymer solutions under stirring.

Characterizations: Dynamic light scattering (DLS) measurements were performed on a laser light scattering spectrometer (BI-200SM) equipped with a digital correlator (BI-9000AT) at $636 \mathrm{~nm}$ at given temperatures. The measurements of the ${ }^{1} \mathrm{H}$ NMR spectra were performed on a varian UNITY plus 400 MHz NMR spectrometer.

\section{RESULTS AND DISCUSSION}

Micellization of the diblock copolymer and TPPS takes place due to the electrostatic interaction between the cationic units of PDMAEMA and negatively charged sulfonate groups of TPPS at $\mathrm{pH}<7$. The formation of complex micelles has a core-shell structure, which was confirmed by ${ }^{1} \mathrm{H}$ NMR analysis (Fig. 2). The intensity of peak a contributed by PNIPAM is almost unchanged in $\mathrm{D}_{2} \mathrm{O}$ at room temperature, because TPPS has little influence on behavior of the PNIPAM block and thus it could be used as a reference value. Spectra A and B were recorded for PDMAEMA- $b$-PNIPAM and complex TPPS/PDMAEMA$b$-PNIPAM in $\mathrm{D}_{2} \mathrm{O}$ at $\mathrm{pH} 2$. The weaken intensity of peak $\mathrm{b}$ contributed by the PDMAEMA block in spectrum B compared to that in spectrum A indicates the mobility of the PDMAEMA block is partially restricted due to the electrostatic interaction with TPPS. Spectrum C was recorded for the complex micelles at $\mathrm{pH}$ 7. The peak b shifts to high region and the intensity of peak b decreases from 0.25 to 0.12 , using the integral intensity of PNIPAM peak a as a reference. These changes suggest that the mobility of the PDMAEMA block is more restricted because of partially deprotonation at $\mathrm{pH}$ close to the $\mathrm{pK}_{\mathrm{a}}$ value. Besides, the typical peaks of TPPS at $\delta=8.84,8.52$ (phenyl protons) and $\delta=9.12$ (pyrrole protons) vanished in the complex micelles (data were not shown). All these results suggest that the complex micelles formed with complex TPPS/PDMAEMA core and PNIPAM shell.

$\mathrm{J}$ - and $\mathrm{H}$-aggregation of TPPS in the micellar core: TPPS shows typical absorption features in different forms. The deprotonated species (free base, $\mathrm{H}_{2} \mathrm{TPPS}^{4-}$ ) exhibits Soret band at $413 \mathrm{~nm}$ and four Q bands at 516, 552, 579 and $633 \mathrm{~nm}$. The zwitterionic diacid species $\left(\mathrm{H}_{4} \mathrm{TPPS}^{2-}, \mathrm{pK}_{\mathrm{a}}-4.9\right)$ shows Soret band at $434 \mathrm{~nm}$ and $\mathrm{Q}$ bands at 594 and $644 \mathrm{~nm}^{12}$.

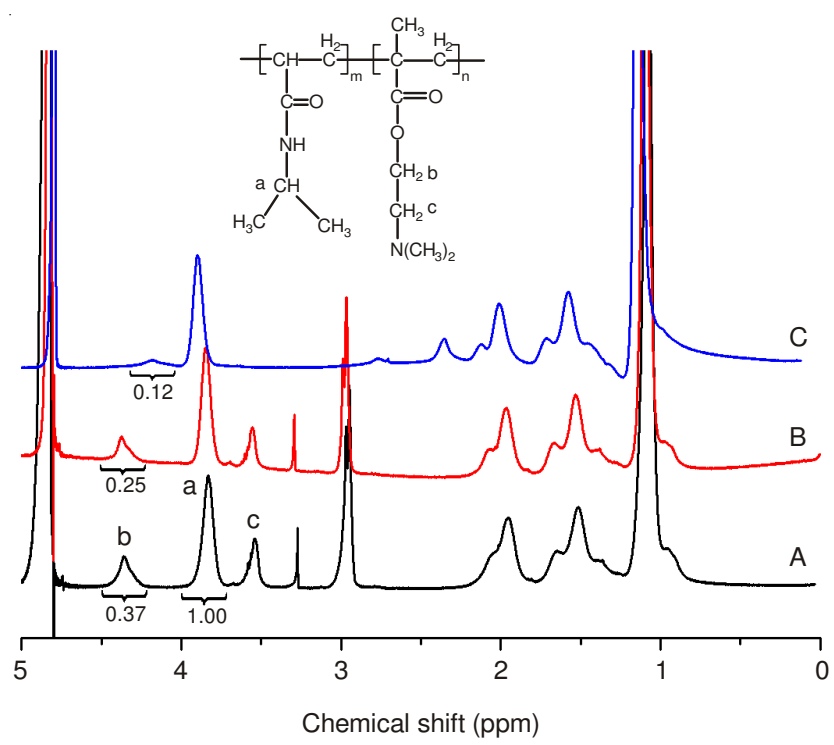

Fig. 2. ${ }^{1} \mathrm{H}$ NMR spectra of the block copolymer at $\mathrm{pH} 2$ (spectrum A), complex micelles at $\mathrm{pH} 2$ (spectrum B) and $\mathrm{pH} 7$ (spectrum C). The spectra were recorded in $\mathrm{D}_{2} \mathrm{O}$ at $25^{\circ} \mathrm{C}$

Aggregation of the dye is also accompanied by spectral shifts due to the excitonic interactions between the chromophores ${ }^{13}$. As shown in Fig. 3, at pH 1.5, the absorption bands of the complex TPPS/PDMAEMA- $b$-PNIPAM appear at 490 and $702 \mathrm{~nm}$, indicating the formation of J-aggregates assembled from diacid porphyrin $\left(\mathrm{H}_{4} \mathrm{TPPS}^{2-}\right)^{14}$. At $\mathrm{pH} 3-7$, the absorptions

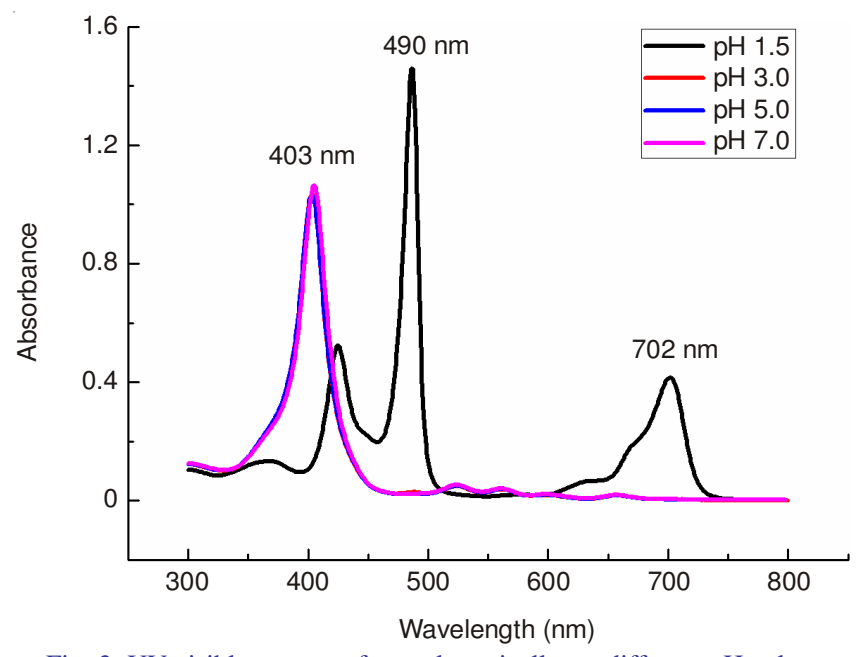

Fig. 3. UV-visible spectra of complex micelles at different $\mathrm{pH}$ values 
at $403 \mathrm{~nm}$ and four red-shift Q bands at 524, 561, 599 and 659 $\mathrm{nm}$ suggests that $\mathrm{H}$-aggregates assembled from free base form of TPPS were dominating species ${ }^{15}$.

J-aggregates are proposed to be stabilized through electrostatic and hydrogen-bonding interactions between the positively charged center of one $\mathrm{H}_{4}$ TPPS $^{2-}$ molecule electrostatic and the negatively charged peripheral sulfonate groups of the adjacent molecules. H-aggregates are resulted from the association of $\pi$ conjugated porphyrin rings. In the micellar core, the anionic TPPS formed J-aggregates at the $\mathrm{pH} 1.5$ and $\mathrm{H}$-aggregates at $\mathrm{pH} 3-7$, respectively. While under the same conditions, TPPS exists as monomer mostly in the absence of the copolymer. It is proved that the electrostatic interaction with PDMAEMA leads to the aggregation. The multiple-point cooperative binding effect of copolymer also favors the aggregation.

Thermo-sensitive of complex micelles: Poly(N-isopropylacrylamide) (PNIPAM) is a well-known thermo-sensitive polymer. When the temperature increases, water progressively becomes a poor solvent for the PNIPAM blocks and thus the stretched PNIPAM chain can collapse on the surface of the hydrophobic core.

To determine the thermo-responsive behavior of the complex micelles, the hydrodynamic diameter $\left(D_{h}\right)$ values of the complex micelles were measured by dynamic light scattering in aqueous solution at different temperatures (Fig. 4). When the temperature is below the LCST of PNIPAM, the micelles form a core-shell structure with TPPS/PDMAEMA as the core and the PNIPAM segment as the shell. Dynamic light scattering data show that the micelles disperse well in water and the $D_{h}$ value is $100 \mathrm{~nm}$ at room temperature. As the temperature increases, the scattering intensity and $D_{h}$ value increase as a result of the inter-micellar aggregation of complex micelles caused by the collapse of PNIPAM. When the temperature is higher than $32{ }^{\circ} \mathrm{C}$, both of the scattering intensity and $\mathrm{D}_{\mathrm{h}}$ value display a sharp growth followed by a gradual increase that begin at $40{ }^{\circ} \mathrm{C}$. The final $\mathrm{D}_{\mathrm{h}}$ value reaches $1000 \mathrm{~nm}$, suggesting a coagulation in the system. That is the stretched PNIPAM chain of the micelles collapses onto the TPPS/PDMAEMA complex core when the temperature is above the LCST of PNIPAM. The LCST of PNIPAM is $31-32{ }^{\circ} \mathrm{C}$, which is similar to the PNIPAM homopolymer.

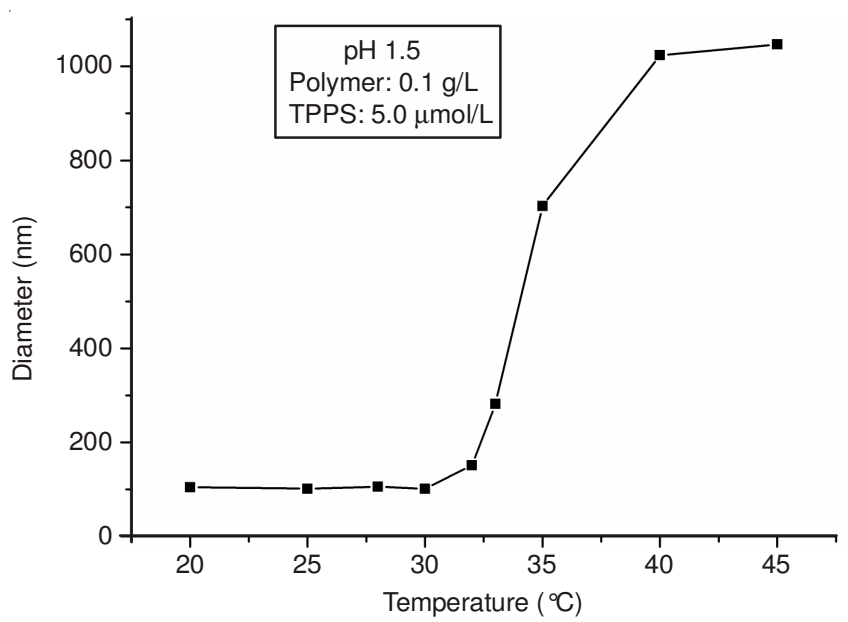

Fig. 4. Hydrodynamic diameters $\left(\mathrm{D}_{\mathrm{h}}\right)$ of complex micelles in solution at different temperatures measured by DLS at the scattering angle of $90^{\circ}$
Despite the thermo-sensitive block caused significant changes in the hydrodynamic diameter of the complex micelles upon temperature increase, the aggregation form of TPPS was not affected. Fig. 5 shows the UV-visible spectra of complex micelles ( $\mathrm{pH}$ 1.5, J-aggregates) at different temperatures. There is no change, but a decrease in the absorption intensity, detected during the temperature increase. The similar phenomena were observed in the case of $\mathrm{H}$-aggregates at $\mathrm{pH}$ 3-7 (data were shown). It is demonstrated that the aggregation form of TPPS is unchanged. The absorption intensity decreases when the temperature is higher than $32{ }^{\circ} \mathrm{C}$, corresponding to the temperature where the sharp increase in scattering light intensity and hydrodynamic diameter happens. Therefore, the thermosensitivity of PNIPAM and the coagulation of micelles lead to the weaken absorption intensity.

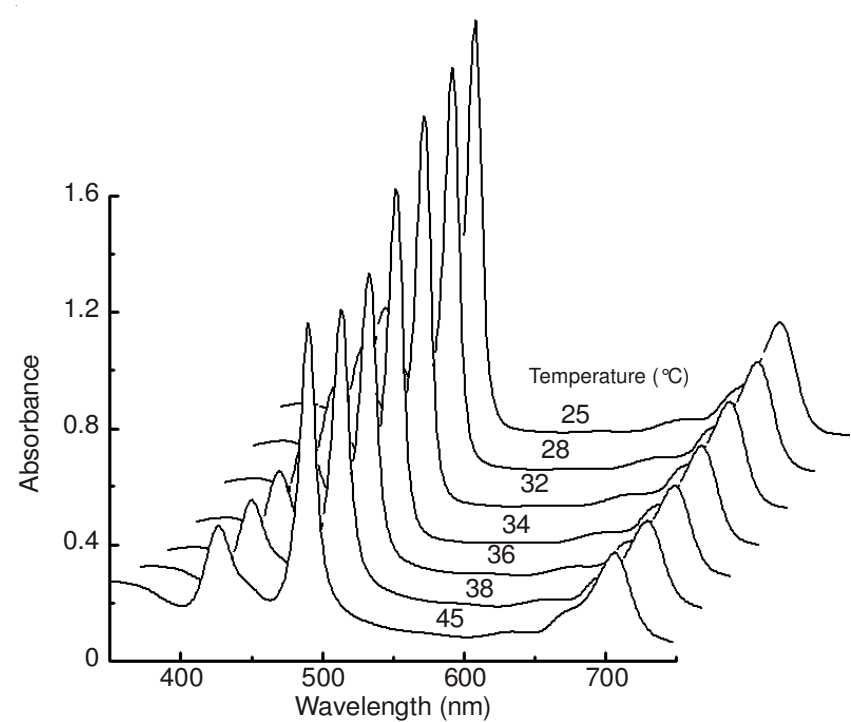

Fig. 5. UV-visible spectra of complex micelles ( $\mathrm{pH} \mathrm{1.5,} \mathrm{J-aggregates)} \mathrm{at}$ different temperatures

\section{Conclusion}

The complex micelles were formed by TPPS and thermoand $\mathrm{pH}$-sensitive block copolymer PDMAEMA- $b$-PNIPAM at $\mathrm{pH}$ below 7 through the electrostatic interaction between the TPPS anions and the PDMAEMA cations. The resultant complex micelles consist of a complex TPPS/PDMAEMA core and a PNIPAM shell at room temperature. TPPS in the micellar core can further assemble into J-aggregates and $\mathrm{H}$-aggregates at $\mathrm{pH}$ 1.5 and 3-7. Thermo-sensitive block PNIPAM can collapse on the complex micellar core and lead to an inter-micellar aggregation, when the temperature was above the lower critical solution temperature (LCST), while the aggregation form of TPPS was not affected by the temperature increase. The studies open a route for a general approach and new pathways to fabricate stimuli responsive nanostructure materials. The $\mathrm{pH}$-induced of aggregation of TPPS may have potential application in molecular device design.

\section{ACKNOWLEDGEMENTS}

This material is based upon work funded by Zhejiang Provincial Natural Science Foundation of China under Grant No. LQ13B070002 and Taizhou City Natural Science Foundation under Grant No. ky102. 


\section{REFERENCES}

1. C.K. Ober, S.Z.D. Cheng, P.T. Hammond, M. Muthukumar, E. Reichmanis, K.L. Wooley and T.P. Lodge, Macromolecules, 42, 465 (2009).

2. F. Bai, Z. Sun, H. Wu, R.E. Haddad, E.N. Coker, J.Y. Huang, M.A. Rodriguez and H. Fan, Nano Lett., 11, 5196 (2011).

3. N.C. Maiti, S. Mazumdar and N. Periasamy, J. Phys. Chem. B, 102, 1528 (1998).

4. S.G. Jiang and M.H. Liu, J. Phys. Chem. B, 108, 2880 (2004).

5. A.S.R. Koti and N. Periasamy, Chem. Mater., 15, 369 (2003).

6. J.F. Gohy, Adv. Polym. Sci., 190, 65 (2005).

7. J. Rao, Z. Luo, Z. Ge, H. Liu and S. Liu, Biomacromolecules, 8, 3871 (2007).

8. L. Zhao, R. Ma, J. Li, Y. Li, Y. An and L. Shi, Biomacromolecules, 9, 2601 (2008).
9. L. Zhao, R. Ma, J. Li, Y. Li, Y. An and L. Shi, Biomacromolecules, 10, 3343 (2009).

10. C. Wu, A. Ying, S. Ren and J. Xu, Asian J. Chem., 25, 3806 (2013).

11. E.B. Fleischer, J.M. Palmer, T.S. Srivastava and A. Chatterjee, J. Am. Chem. Soc., 93, 3162 (1971).

12. G. De Luca, A. Romeo and L.M. Scolaro, J. Phys. Chem. B, 110, 7309 (2006).

13. N.C. Maiti, S. Mazumdar and N. Periasamy, J. Porphyr. Phthalocyanines, 2, 369 (1998).

14. A.S.R. Koti, J. Taneja and N. Periasamy, Chem. Phys. Lett., 375, 171 (2003).

15. M.A. Castriciano, A. Romeo, V. Villari, N. Angelini, N. Micali and L.M. Scolaro, J. Phys. Chem. B, 109, 12086 (2005). 